\title{
CONVERGENCE OF THE NONCONFORMING WILSON ELEMENT FOR A CLASS OF NONLINEAR PARABOLIC PROBLEMS
}

\author{
S. H. CHOU AND Q. LI
}

\begin{abstract}
This paper deals with the convergence properties of the nonconforming quadrilateral Wilson element for a class of nonlinear parabolic problems in two space dimensions. Optimal $H^{1}$ and $L_{2}$ error estimates for the continuous time Galerkin approximations are derived. It is also shown for rectangular meshes that the gradient of the Wilson element solution possesses superconvergence, and that the $L_{\infty}$ error on the gradient is of order $h \log (1 / h)$.
\end{abstract}

\section{INTRODUCTION}

The need to reduce the computational work in the use of conforming elements for the solution of higher-order elliptic problems has led to the invention of nonconforming elements. An issue in the study of nonconforming elements is the creation of a proper test that guarantees convergence of the elements. Recently, Shi [3] gave a certain condition on mesh subdivisions which ensures convergence of the quadrilateral Wilson element applied to a class of elliptic problems. The basic error analysis of Galerkin methods applied to parabolic equations is studied in $[1,6]$, where conforming finite elements are considered. In this paper we extend the techniques and results in $[1,6]$ to the nonconforming quadrilateral Wilson element under the aforementioned condition in [3]. It is found that the convergence rates of approximate solutions for the problems under consideration have the same order of accuracy as in the comparable elliptic problems. For the nonconforming rectangular Wilson element, the $L_{\infty}$ error estimate and superconvergence for the gradient are shown as well. The paper is organized as follows. Section 2 deals with a general description of nonconforming methods for solving nonlinear parabolic problems. Section 3 contains some lemmas concerning error estimates for an auxiliary variational problem. The main results of the paper are established in $\S 4$. Optimal $L_{2}$ and $H^{1}$ error estimates, as well as almost optimal maximum-norm error and superconvergence order estimates for the gradient, are obtained.

Received October 20, 1988.

1980 Mathematics Subject Classification (1985 Revision). Primary 65N30.

Key words and phrases. Parabolic equation, Wilson element. 


\section{NONCONFORMING METHODS FOR SOLVING NONLINEAR PARABOLIC PROBLEMS}

Let $\Omega$ be a polygonal domain in $R^{2}$ with boundary $\partial \Omega$. Consider the following nonlinear parabolic initial-boundary value problem:

$$
\begin{array}{ll}
u_{t}-\nabla \cdot(a(x, u) \nabla u)=\sum_{i=1}^{2} b_{i}(x, u) u_{x_{i}}+f(x, u) & \text { in } \Omega \times I, \\
u=0 & \text { on } \partial \Omega \times I, \\
u(x, 0)=u_{0}(x) & \text { in } \Omega,
\end{array}
$$

where $I$ denotes a finite interval $[0, T]$.

We shall assume that $a, b_{i}$, and $f$ are sufficiently smooth functions on $\bar{\Omega} \times R$ which, together with an appropriate number of derivatives, are bounded there, and that the function $a$ satisfies

$$
0<c_{1} \leq a(x, u) \leq c_{2} \text { for }(x, u) \in \bar{\Omega} \times R,
$$

for some constants $c_{1}$ and $c_{2}$. The global nature of our assumptions with respect to $u$ does not constitute any serious restriction. In fact, the approximate solutions to be considered will be shown to be uniformly close to the exact solution $u$, and thus depend only on the nature of $a, b_{i}$, and $f$ in a neighborhood of the range of $u$.

Let $W^{s, p}(G), 1 \leq p \leq \infty, H^{s}(G)=W^{s, 2}(G)$, and $H^{s}(\partial G), s \in R$, be the usual Sobolev spaces on $G$ and $\partial G$. The associated norms and seminorms are denoted as follows:

$$
\begin{array}{cl}
\|\cdot\|_{s, p, G}=\|\cdot\|_{W^{s, p}(G)}, & \|\cdot\|_{s, G}=\|\cdot\|_{H^{s}(G)}, \\
\|\cdot\|_{s, \partial G}=\|\cdot\|_{H^{s}(\partial G)}, & \|\cdot\|_{s, p}=\|\cdot\|_{W^{s, p}(\Omega)}, \\
\|\cdot\|_{s}=\|\cdot\|_{H^{s}(\Omega)}, \quad\|\cdot\|=\|\cdot\|_{L_{2}(\Omega)}, \\
|\cdot|_{s, G}=|\cdot|_{H^{s}(G)}, \quad|\cdot|_{s}=|\cdot|_{H^{s}(\Omega)}, \quad\|\cdot\|_{L_{\infty}}=\|\cdot\|_{L_{\infty}(\Omega)} .
\end{array}
$$

Let $X$ be a normed vector space with norm $\|\cdot\|_{X}$. For $\varphi: I \rightarrow X$, define

$$
\|\varphi\|_{L_{2}(X)}^{2}=\int_{0}^{T}\|\varphi(t)\|_{X}^{2} d t, \quad\|\varphi\|_{L_{\infty}(X)}=\sup _{t \in I}\|\varphi(t)\|_{X} .
$$

We now consider the numerical approximation of (2.1) by a nonconforming finite element method. Let $\bar{\Omega}=\bigcup_{K \in K_{h}} K$ be a decomposition of $\bar{\Omega}$ into elements $K$ with diameters $\leq h$. With this subdivision we associate a nonconforming finite element space $S_{h}$ consisting of certain functions $v$ vanishing at the nodes belonging to $\partial \Omega$. In general, the inclusion $S_{h} \subset H_{0}^{1}(\Omega)$ does not hold. The norm associated with $S_{h}$ is

$$
\|\cdot\|_{S_{h}}^{2}=|\cdot|_{1, h}^{2}=\sum_{K}|\cdot|_{1, K}^{2} .
$$

In this paper we shall confine ourselves to the nonconforming spaces $S_{h}$ of the Wilson element, i.e., the finite element spaces of all functions whose restrictions to each quadrilateral element $K \in K_{h}$ are the shape functions defined by equations (3.1)-(3.3) of [3]. We also assume the subdivisions $K_{h}$ to satisfy the 
regularity conditions in [3]. Let $h_{K}$ denote the diameter of the element $K$ and $h=\max _{K \in K_{h}} h_{K}$. We introduce a further assumption on mesh subdivisions. The distance $d_{K}$ between the midpoints of the diagonals of $K \in K_{h}$ is of the order $O\left(h_{K}^{2}\right)$, uniformly for all elements $K$ as $h \rightarrow 0$.

The nonconforming approximation of the solution of $(2.1)$ is the following. Find a differentiable map $U: I \rightarrow S_{h}$ such that

$$
\begin{aligned}
\left(U_{t}, V\right)+A_{h}(U ; U, V) & =\sum_{i=1}^{2}\left(b_{i}(U) U_{x_{i}}, V\right)_{h}+(f(U), V), \\
(U(0), V) & =\left(u_{0}, V\right) \quad \forall V \in S_{h},
\end{aligned}
$$

where

$$
\begin{gathered}
(\varphi, \psi)=\int_{\Omega} \varphi \psi d x, \quad(\varphi, \psi)_{h}=\sum_{K} \int_{K} \varphi \psi d x, \\
A_{h}(p ; \varphi, \psi)=(a(p) \nabla \varphi, \nabla \psi)_{h}=\sum_{K}(a(p) \nabla \varphi, \nabla \psi)_{K}, \\
a(p)=a(x, p), \quad f(U)=f(x, U), \quad U(0)=U(x, 0) .
\end{gathered}
$$

Here, $(\cdot, \cdot)_{K}$ denotes the inner product in $L_{2}(K)^{2}$. Without loss of generality we shall assume the initial condition in $(2.1)$ to be $u_{0}(x)=0$ throughout this paper.

\section{LEMMAS}

Let $u$ and $U$ be the solutions of problems (2.1) and (2.3), respectively. In order to estimate the error $U-u$, we introduce an auxiliary variational problem:

Find a map $\tilde{u}: I \rightarrow S_{h}$ such that

$$
A_{h}(u ; \tilde{u}, V)=A(u ; u, V) \quad \forall V \in S_{h},
$$

where

$$
A(w, u, V)=-\int_{\Omega} \nabla \cdot(a(w) \nabla u) V d x .
$$

Note that the initial condition $\tilde{u}(0)=0$ holds, since $u_{0}(x)=0$. In the sequel, $c$ will be used as a generic constant. It may have different values at different places.

Lemma 3.1. Assume $\psi \in H^{1}(\Omega)$ and $v_{h} \in S_{h}$; then

$$
\left|T_{h}^{(r)}\left(\psi, v_{h}\right)\right|:=\left|\sum_{K} \int_{\partial K} \psi v_{h} N_{r} d s\right| \leq c h^{2}\|\psi\|_{1}\left|v_{h}\right|_{2, h},
$$

where $n=\left(N_{1}, N_{2}\right)$ is the unit exterior normal vector along the boundary $\partial K$ of $K$, and the seminorm $|\cdot|_{2, h}$ is defined by $|\cdot|_{2, h}^{2}=\sum_{K}|\cdot|_{2, K}^{2}$.

Proof. The proof uses ideas contained in [3, Theorem 1]. We decompose the trial function $v_{h}$ into the conforming and nonconforming parts, $v_{h}=y_{h}+z_{h}$. Since $y_{h} \in H_{0}^{1}(\Omega)$,

$$
T_{h}^{(r)}\left(\psi, y_{h}\right)=0, \quad T_{h}^{(r)}\left(\psi, v_{h}\right)=T_{h}^{(r)}\left(\psi, z_{h}\right) .
$$


Let us decompose $T_{h}^{(r)}\left(\psi, z_{h}\right)$ in the form

$$
\begin{aligned}
T_{h}^{(r)}\left(\psi, z_{h}\right)= & \sum_{K} \int_{\partial K} \psi z_{h} N_{r} d s \\
= & \sum_{K} \int_{\partial K} R_{0} \psi R_{0} z_{h} N_{r} d s+\sum_{K} \int_{\partial K} R_{0} \psi P_{0} z_{h} N_{r} d s \\
& +\sum_{K} \int_{\partial K} P_{0} \psi z_{h} N_{r} d s=\sum_{j=1}^{3} T_{j},
\end{aligned}
$$

where $P_{0} v$ is the piecewise constant approximation of $v$ defined in [3, equation (2.6)] and $R_{0} v=v-P_{0} v$ is the associated remainder term. We estimate each of the terms $T_{i}, i=1,2,3$, on the right-hand side of (3.4).

(i) By virtue of interpolation theory we have

$$
\begin{aligned}
\left|T_{1}\right| & \leq \sum_{K}\left|\int_{\partial K} R_{0} \psi R_{0} z_{h} N_{r} d s\right| \\
& \leq \sum_{K}\left(\int_{\partial K}\left(R_{0} \psi\right)^{2} d s\right)^{1 / 2}\left(\int_{\partial K}\left(R_{0} z_{h}\right)^{2} d s\right)^{1 / 2} \\
& \leq c h|\psi|_{1}\left|z_{h}\right|_{1, h} .
\end{aligned}
$$

Combining [3, (3.11)-(3.12); 2, (2.11); and 3, (2.3)] yields

$$
\left|z_{h}\right|_{1, K} \leq c h_{K}\left|v_{h}\right|_{2, K} \text {. }
$$

Consequently,

$$
\left|T_{1}\right| \leq \operatorname{ch}^{2}|\psi|_{1}\left|v_{h}\right|_{2, h} .
$$

(ii) Similarly, we have

$$
\left|P_{0} z_{h}\right| \leq c h_{K}\left|v_{h}\right|_{2, K} .
$$

Thus,

$$
\begin{aligned}
\left|T_{2}\right| & \leq \sum_{K}\left|\int_{\partial K} R_{0} \psi P_{0} z_{h} N_{r} d s\right| \\
& \leq \sum_{K}\left(\int_{\partial K}\left(R_{0} \psi\right)^{2} d s\right)^{1 / 2}\left(\int_{\partial K}\left(P_{0} z_{h}\right)^{2} d s\right)^{1 / 2} \\
& \leq \operatorname{ch}^{2}|\psi|_{1}\left|v_{h}\right|_{2, h} .
\end{aligned}
$$

(iii) For the term $T_{3}$, we use the inequalities [3, (3.17)-(3.20) and (3.5)] and note that $d_{K}=O\left(h_{K}^{2}\right)$ to get

$$
\left|\int_{\partial K} z_{h} N_{r} d s\right| \leq c d_{K} h_{K}\left|v_{h}\right|_{2, K} \leq c h^{3}\left|v_{h}\right|_{2, K} .
$$

Thus,

$$
\left|T_{3}\right| \leq \sum_{K}\left|\int_{\partial K} P_{0} \psi z_{h} N_{r} d s\right| \leq c h^{2}\|\psi\|_{1}\left|v_{h}\right|_{2, h} .
$$


Combining relations $(3.3),(3.4),(3.6),(3.8)$, and (3.10), we see that $(3.2)$ follows.

Corollary 3.1. For $\psi \in H^{1}(\Omega)$ and $v_{h} \in S_{h}$,

$$
\left|T_{h}^{(r)}\left(\psi, v_{h}\right)\right| \leq \operatorname{ch}\|\psi\|_{1}\left|v_{h}\right|_{1, h}, \quad r=1,2 .
$$

The following three lemmas are contained in [3, 4, and 5], respectively.

Lemma 3.2. Let $u \in H^{2}(\Omega)$ for all $t \in I$ and $\tilde{u}$ be the solutions of (2.1) and (3.1), respectively. Then for $t \in I$

$$
\|\tilde{u}-u\|+h|\tilde{u}-u|_{1, h} \leq c h^{2}\|u\|_{2} .
$$

Lemma 3.3. Under the hypotheses of Lemma 3.2 and the additional assumption that $\bar{\Omega}$ is decomposed into rectangular elements, we have that

$$
u \in W^{2, \infty}(\Omega) \quad \forall t \in I
$$

implies

$$
\|\tilde{u}-u\|_{L_{\infty}} \leq c h^{2} \log \frac{1}{h}\|u\|_{2, \infty}
$$

Lemma 3.4. If, in addition to the hypotheses of Lemma 3.3, $u \in H^{3}(\Omega)$ for $t \in I$, then for $t \in I$

$$
\left\{\frac{1}{N} \sum_{x_{0} \in G}\left|\nabla(\tilde{u}-u)\left(x_{0}\right)\right|^{2}\right\}^{1 / 2} \leq c h^{2}\|u\|_{3},
$$

where $G$ denotes the set of nice stress points and $N=O\left(h^{-2}\right)$ is the cardinal number of $G$.

(The nice stress points relative to the given subdivision are the centers of the rectangular elements of that subdivision.)

We now give the following estimates for the error $\tilde{u}-u$ between solutions of (3.1) and (2.1).

Lemma 3.5. Under the hypotheses of Lemma 3.2, we have for $t \in I$

$$
\begin{gathered}
\left(\sum_{K}\|\tilde{u}-u\|_{-1 / 2, \partial K}^{2}\right)^{1 / 2} \leq c h^{2}\|u\|_{2}, \\
\left(\sum_{K}\left\|\tilde{u}_{t}-u_{t}\right\|_{-1 / 2, \partial K}\right)^{1 / 2} \leq c h^{2}\left(\|u\|_{2}+\left\|u_{t}\right\|_{2}\right) .
\end{gathered}
$$

Proof. (i) Let $\varphi_{K} \in H^{1}(K)$ be the solution of the elliptic problem

$$
\int_{K} a(u) \nabla \varphi_{K} \cdot \nabla v d x=\int_{\partial K} \gamma_{K} v d s \quad \forall v \in H^{1}(K), t \in I .
$$


Then $\gamma_{K} \in H^{1 / 2}(\partial K)$ may be chosen so that

$$
\int_{\partial K} \gamma_{K} \eta d s=\|\eta\|_{-1 / 2, \partial K}^{2}
$$

and

$$
\left\|\gamma_{K}\right\|_{1 / 2, \partial K}=\|\eta\|_{-1 / 2, \partial K},
$$

where $\eta=\tilde{u}-u$. The existence of $\gamma_{K}$ is guaranteed by the Hahn-Banach theorem, since $H^{1 / 2}(\partial K)$ is the dual of $H^{-1 / 2}(\partial K)$.

It is well known that if $K$ is convex, then $\varphi_{K} \in H^{2}(K)$ and

$$
\left\|\varphi_{K}\right\|_{2, K} \leq c\left\|\gamma_{K}\right\|_{1 / 2, \partial K} \leq c\|\eta\|_{-1 / 2, \partial K} .
$$

Setting $v=\eta$ in (3.17) and summing over $K \in K_{h}$, we find

$$
\sum_{K}\|\eta\|_{-1 / 2, \partial K}^{2}=A_{h}(u ; \eta, \varphi)=A_{h}(u ; \eta, \varphi-\pi \varphi)+A_{h}(u ; \eta, \pi \varphi),
$$

where the function $\varphi$ is defined by $\left.\varphi\right|_{K}=\varphi_{K}$ and $\pi \varphi \in S_{h}$ denotes the $S_{h}$ interpolant of $\varphi$. By the continuity of $A_{h}(u ; \cdot, \cdot)$, the interpolation property, Lemma 3.2, and (3.18), we get

$$
\begin{aligned}
\left|A_{h}(u ; \eta, \varphi-\pi \varphi)\right| & \leq c|\eta|_{1, h}|\varphi-\pi \varphi|_{1, h} \leq c h^{2}\|u\|_{2}|\varphi|_{2, h} \\
& \leq c h^{2}\|u\|_{2}\left(\sum_{K}\|\eta\|_{-1 / 2, \partial K}^{2}\right)^{1 / 2} .
\end{aligned}
$$

For the second term on the right-hand side of (3.19), we have, by (3.1),

$$
\begin{aligned}
A_{h}(u ; \eta, \pi \varphi) & =A_{h}(u ; \tilde{u}, \pi \varphi)-A_{h}(u ; u, \pi \varphi) \\
& =A(u ; u, \pi \varphi)-A_{h}(u ; u, \pi \varphi)=-D_{h}(u ; u, \pi \varphi),
\end{aligned}
$$

where

$$
\begin{aligned}
D_{h}\left(w ; u, v_{h}\right) & =A_{h}\left(w ; u, v_{h}\right)-A\left(w ; u, v_{h}\right) \\
& =\sum_{K} \int_{K}\left[a(w) \nabla u \cdot \nabla v_{h}+\nabla \cdot(a(w) \nabla u) v_{h}\right] d x \\
& =\sum_{K} D_{K}\left(w ; u, v_{h}\right) .
\end{aligned}
$$

Using Green's formula, we obtain

$$
D_{K}(w ; u, v)=\int_{\partial K} a(w) \frac{\partial u}{\partial n} v_{h} d s .
$$

It follows from Lemma 3.1 and (3.18) that

$$
\begin{aligned}
\left|A_{h}(u ; \eta, \pi \varphi)\right| & \leq c h^{2}\|u\|_{2}|\pi \varphi|_{2, h} \leq c h^{2}\|u\|_{2}|\varphi|_{2, h} \\
& \leq c h^{2}\|u\|_{2}\left(\sum_{K}\|\eta\|_{-1 / 2, \dot{\alpha} K}^{2}\right)^{1 / 2} .
\end{aligned}
$$

Inequality (3.15) now follows from (3.19) and inequalities (3.20) and (3.24). 
(ii) The second assertion of the lemma can be proven using the same technique as the one leading to (3.15).

Similarly as (3.19) was derived, one can easily obtain

$$
\sum_{K}\left\|\eta_{t}\right\|_{-1 / 2, \partial K}^{2}=A_{h}\left(u ; \eta_{t}, \varphi-\pi \varphi\right)+A_{h}\left(u ; \eta_{t}, \pi \varphi\right),
$$

where

$$
\|\varphi\|_{2, K} \leq c\left\|\eta_{t}\right\|_{-1 / 2, \partial K}
$$

Obviously,

$$
\left|A_{h}\left(u ; \eta_{t}, \varphi-\pi \varphi\right)\right| \leq c h^{2}\|u\|_{2}\left(\sum_{K}\left\|\eta_{t}\right\|_{-1 / 2, \partial K}^{2}\right)^{1 / 2} .
$$

To estimate the second term on the right-hand side of (3.25), we rewrite the representation (3.1) as

$$
A_{h}(u ; \eta, V)+D_{h}(u ; u, V)=0 \quad \forall V \in S_{h} .
$$

Differentiating (3.28) with respect to $t$, we see that

$$
A_{h}\left(u ; \eta_{t}, V\right)+A_{h}^{*}(u ; \eta, V)+D_{h}\left(u ; u_{t}, V\right)+D_{h}^{*}(u ; u, V)=0 \quad \forall V \in S_{h},
$$

where

$$
\begin{aligned}
& A_{h}^{*}(w ; v, V)=\sum_{K} \int_{K}(a(w))_{t} \nabla v \cdot \nabla V d x \\
& D_{h}^{*}(w ; v, V)=\sum_{K} \int_{\partial K}(a(w))_{t} \frac{\partial v}{\partial n} V d s .
\end{aligned}
$$

Hence,

$$
A_{h}\left(u ; \eta_{t}, \pi \varphi\right)=-D_{h}\left(u ; u_{t}, \pi \varphi\right)-D_{h}^{*}(u ; u, \pi \varphi)-A_{h}^{*}(u ; \eta, \pi \varphi) .
$$

Applying Lemma 3.1 to the first two terms on the right-hand side of (3.32), we conclude that

$$
\begin{aligned}
\left|D_{h}\left(u ; u_{t}, \pi \varphi\right)\right| & \leq c h^{2}\left\|u_{t}\right\|_{2}\|\varphi\|_{2, h}, \\
\left|D_{h}^{*}(u ; u, \pi \varphi)\right| & \leq c h^{2}\|u\|_{2}\|\varphi\|_{2, h} .
\end{aligned}
$$

For the last term of (3.32), we have by Green's formula,

$$
\begin{aligned}
-A_{h}^{*}(u ; \eta, \pi \varphi) & =\sum_{K} \int_{K} \nabla \cdot\left(a(u)_{t} \nabla \pi \varphi\right) \eta d x-\sum_{K} \int_{\partial K}(a(u))_{t} \eta \frac{\partial \pi \varphi}{\partial n} d x \\
& =G_{1}+G_{2},
\end{aligned}
$$

and consequently, writing $\|\cdot\|_{2, h}^{2}=\|\cdot\|^{2}+|\cdot|_{1, h}^{2}+|\cdot|_{2, h}^{2}$,

$$
\left|G_{1}\right| \leq c\|\eta\|\|\pi \varphi\|_{2, h} \leq c h^{2}\|u\|_{2}\|\varphi\|_{2, h},
$$




$$
\left|G_{2}\right| \leq c \sum_{K}\|\eta\|_{-1 / 2, \partial K}\left\|\frac{\partial \pi \varphi}{\partial n}\right\|_{1 / 2, \partial K} \leq c\left(\sum_{K}\|\eta\|_{-1 / 2, \partial K}^{2}\right)^{1 / 2}\|\varphi\|_{2, h},
$$

where we have used the trace inequality. Combining the inequalities (3.26), (3.33)-(3.37), and (3.15), we see from (3.32) that

$$
\left|A_{h}\left(u ; \eta_{t}, \pi \varphi\right)\right| \leq c h^{2}\|u\|_{2}\left(\sum_{K}\left\|\eta_{t}\right\|_{-1 / 2, \partial K}^{2}\right)^{1 / 2} .
$$

Finally, substituting (3.27) and (3.38) into (3.25), we arrive at (3.16).

Lemma 3.6. Assume that the hypotheses of Lemma 3.2 are satisfied. Moreover, assume that $u_{t}, u_{t t} \in H^{2}(\Omega)$ for $t \in I$; then, for $t \in I$,

$$
\begin{gathered}
\left\|\tilde{u}_{t}-u_{t}\right\|+h\left|\tilde{u}_{t}-u_{t}\right|_{1, h} \leq c h^{2}\left[\|u\|_{2}+\left\|u_{t}\right\|_{2}\right], \\
\left\|\tilde{u}_{t t}-u_{t t}\right\|+h\left|\tilde{u}_{t t}-u_{t t}\right|_{1, h} \leq c h^{2}\left[\|u\|_{2}+\left\|u_{t}\right\|_{2}+\left\|u_{t t}\right\|_{2}\right] .
\end{gathered}
$$

Proof. (i) Let $u^{*} \in S_{h}$ be such that for $t \in I$

$$
A_{h}\left(u ; u^{*}-u_{t}, V\right)+D_{h}\left(u ; u_{t}, V\right)=0 \quad \forall V \in S_{h} .
$$

Then by Lemma 3.2,

$$
\left|u^{*}-u_{t}\right|_{1, h} \leq c h\left\|u_{t}\right\|_{2} .
$$

Using inequality (2.2) and Corollary 3.1, we deduce from (3.29) and (3.41) with $V=\tilde{u}_{t}-u^{*}$ that

$$
\begin{aligned}
c_{1}\left|\tilde{u}_{t}-u^{*}\right|_{1, h}^{2} & \leq A_{h}\left(u ; \tilde{u}_{t}-u^{*}, \tilde{u}_{t}-u^{*}\right) \\
& =-D^{*}\left(u ; u, \tilde{u}_{t}-u^{*}\right)-A_{h}^{*}\left(u ; \tilde{u}-u, \tilde{u}_{t}-u^{*}\right) \\
& \leq c\left[h\|u\|_{2}+|\tilde{u}-u|_{1, h}\right]\left|\tilde{u}_{t}-u^{*}\right|_{1, h} \leq c h\|u\|_{2}\left|\tilde{u}_{t}-u^{*}\right|_{1, h} .
\end{aligned}
$$

Combining this inequality with the triangular inequality

$$
\left|\tilde{u}_{t}-u_{t}\right|_{1, h} \leq\left|\tilde{u}_{t}-u^{*}\right|_{1, h}+\left|u^{*}-u_{t}\right|_{1, h}
$$

and using (3.42) yield

$$
\left|\tilde{u}_{t}-u_{t}\right|_{1, h} \leq \operatorname{ch}\left[\|u\|_{2}+\left\|u_{t}\right\|_{2}\right] .
$$

For the $L_{2}$-norm we proceed by duality. Note that

$$
\left\|\eta_{t}\right\|=\sup _{g \in L_{2}} \frac{\left|\left(g, \eta_{t}\right)\right|}{\|g\|} .
$$

Let $g$ be an arbitrary function in $L_{2}(\Omega)$. Let $\varphi \in H^{2}(\Omega) \cap H_{0}^{1}(\Omega)$ be the solution of

$$
\begin{aligned}
-\nabla \cdot(a(u) \nabla \varphi) & =g & & \text { in } \Omega, \\
\varphi & =0 & & \text { on } \partial \Omega .
\end{aligned}
$$

Recall that

$$
\|\varphi\|_{2} \leq c\|g\| .
$$


From (3.29) we have

$$
\begin{aligned}
\left(g, \eta_{t}\right)= & A_{h}\left(u ; \eta_{t}, \varphi-\pi \varphi\right)-A_{h}^{*}(u ; \eta, \pi \varphi)-D_{h}\left(u ; u_{t}, \pi \varphi\right) \\
& -D_{h}^{*}(u ; u, \pi \varphi)-\left[A_{h}\left(u ; \eta_{t}, \varphi\right)-\left(g, \eta_{t}\right)\right] \\
= & \sum_{j=1}^{5} I_{j} .
\end{aligned}
$$

Using arguments similar to those used to derive inequalities (3.27) and (3.38), one has

$$
I_{1}+I_{2}+I_{3}+I_{4} \leq c h^{2}\|u\|_{2}\|\varphi\|_{2} .
$$

Noting that $u_{t} \in H_{0}^{1}(\Omega)$, we have from (3.45) and (3.22) that

$$
I_{5}=-D_{h}(u ; \varphi, \eta)=-D_{h}\left(u ; \varphi, \tilde{u}_{t}\right),
$$

and

$$
\left|I_{5}\right| \leq c h^{2}\|\varphi\|_{2}\left|\tilde{u}_{t}\right|_{2, h} .
$$

The inverse inequality implies

$$
\begin{aligned}
\left|\tilde{u}_{t}\right|_{2, h} & \leq\left|\tilde{u}_{t}-\Pi u_{t}\right|_{2, h}+\left|\Pi u_{t}\right|_{2, h} \\
& \leq c h^{-1}\left[\left|\tilde{u}_{t}-u_{t}\right|_{1, h}+\left|u_{t}-\Pi u_{t}\right|_{1, h}\right]+\left|\Pi u_{t}-u_{t}\right|_{2, h}+\left|u_{t}\right|_{2} \\
& \leq c\left\|u_{t}\right\|_{2} .
\end{aligned}
$$

Together with (3.44) and (3.46)-(3.49), this proves the first estimate of the lemma.

(ii) We now prove (3.40). Differentiating (3.29) with respect to $t$, we get

$$
\begin{gathered}
A_{h}\left(u ; \eta_{t t}, V\right)+2 A_{h}^{*}\left(u ; \eta_{t}, V\right)+A_{h}^{* *}(u ; \eta, V)+D_{h}\left(u ; u_{t t}, V\right) \\
+2 D_{h}^{*}\left(u ; u_{t}, V\right)+D_{h}^{* *}(u ; u, V)=0 \quad \forall V \in S_{h},
\end{gathered}
$$

where

$$
\begin{aligned}
& A_{h}^{* *}(u ; \eta, V)=\sum_{K} \int_{K}(a(u))_{t t} \nabla \eta \cdot \nabla V d x, \\
& D_{h}^{* *}(u ; u, V)=\sum_{K} \int_{\partial K}(a(u))_{t t} \frac{\partial u}{\partial n} V d s .
\end{aligned}
$$

Define $u^{* *} \in S_{h}$ by

$$
A_{h}\left(u ; u^{* *}-u_{t t}, V\right)+D_{h}\left(u ; u_{t t}, V\right)=0 \quad \forall V \in S_{h}, t \in I .
$$

Observing

$$
\left|u^{* *}-u_{t t}\right|_{1, h} \leq c h\left\|u_{t t}\right\|_{2},
$$

and setting $V=\tilde{u}_{t t}-u^{* *}$ in (3.50), we find

$$
\begin{aligned}
c_{1}\left|\tilde{u}_{t t}-u^{* *}\right|_{1, h}^{2} \leq & A_{h}\left(u ; \tilde{u}_{t t}-u^{* *}, \tilde{u}_{t t}-u^{* *}\right) \\
\leq & -2 D_{h}^{*}\left(u ; u_{t}, \tilde{u}_{t t}-u^{* *}\right)-D_{h}^{* *}\left(u ; u, \tilde{u}_{t t}-u^{* *}\right) \\
& -2 A_{h}^{*}\left(u ; \eta_{t}, \tilde{u}_{t t}-u^{* *}\right)-A_{h}^{* *}\left(u ; \eta, \tilde{u}_{t t}-u^{* *}\right) .
\end{aligned}
$$


On the other hand, again by (3.45), it follows from (3.50) that

$$
\begin{aligned}
\left(g, \eta_{t t}\right)= & A_{h}\left(u ; \eta_{t t}, \varphi-\pi \varphi\right)-2 A_{h}^{*}\left(u ; \eta_{t}, \pi \varphi\right)-A_{h}^{* *}(u ; \eta, \pi \varphi) \\
& -D_{h}\left(u ; u_{t t}, \pi \varphi\right)-2 D_{h}^{*}\left(u ; u_{t}, \pi \varphi\right) \\
& -D_{h}^{* *}(u ; u, \pi \varphi)-D_{h}\left(u ; \varphi, \eta_{t t}\right) .
\end{aligned}
$$

Applying the technique used to estimate (3.43), (3.47)-(3.52), and (3.53), inequality (3.40) follows.

Corollary 3.2. Assume that $u, u_{t} \in H^{2}(\Omega) \cap W^{1, \infty}(\Omega)$ for $t \in I$. Then $\nabla \tilde{u}$, $\tilde{u}_{t}$, and $\nabla \tilde{u}_{t}$ are uniformly bounded, i.e., there exists a constant $\tilde{c}$ independent of $h$ and $t$ such that

$$
\|\nabla \tilde{u}\|_{L_{\infty}\left(L_{\infty}\right)}+\left\|\tilde{u}_{t}\right\|_{L_{\infty}\left(L_{\infty}\right)}+\left\|\nabla \tilde{u}_{t}\right\|_{L_{\infty}\left(L_{\infty}\right)} \leq \tilde{c}
$$

where $\|\nabla \varphi\|_{L_{\infty}\left(L_{\infty}\right)}=\sup _{t \in I} \max _{K}\|\nabla \varphi\|_{L_{\infty}(K)}$.

Proof. Using the triangle inequality and the inverse inequality, we have

$$
\begin{aligned}
\|\nabla \tilde{u}\|_{L_{\infty}} & \leq\|\nabla(\tilde{u}-\Pi u)\|_{L_{\infty}}+\|\nabla(\Pi u-u)\|_{L_{\infty}}+\|\nabla u\|_{L_{\infty}} \\
& \leq c h^{-1}\left[|\tilde{u}-u|_{1, h}+|u-\Pi u|_{1, h}\right]+\|\nabla(\pi u-u)\|_{L_{\infty}}+\|\nabla u\|_{L_{\infty}} \\
& \leq c\left[\|u\|_{2}+\|\nabla u\|_{L_{\infty}}\right]+\|\nabla(u-\pi u)\|_{L_{\infty}} .
\end{aligned}
$$

We now estimate the last term of (3.55). Since $\pi u \in S_{h}$, we can write $\pi u$ as the sum of the conforming part $y_{h}$ and the nonconforming part $z_{h}$,

$$
\pi u=y_{h}+z_{h} \text {. }
$$

Hence,

$$
\begin{aligned}
\|\nabla(u-\Pi u)\|_{L_{\infty}} & \leq\left\|\nabla u-y_{h}\right\|_{L_{\infty}}+\left\|\nabla z_{h}\right\|_{L_{\infty}} \\
& \leq\left\|\nabla\left(u-y_{h}\right)\right\|_{L_{\infty}}+c h^{-1}\left|z_{h}\right|_{1, h} \\
& \leq\left\|\nabla\left(u-y_{h}\right)\right\|_{L_{\infty}}+c h^{-1}\left[|\pi u-u|_{1, h}+\left|u-y_{h}\right|_{1}\right] \\
& \leq c\left[\|\nabla u\|_{L_{\infty}}+\|u\|_{2}\right] .
\end{aligned}
$$

Together with (3.55), these bounds show our assertions on $\nabla \tilde{u}$. In the same way, the uniform boundedness of $\tilde{u}_{t}$ and $\nabla \tilde{u}_{t}$ can be proved. We omit the details.

\section{ERROR ESTIMATES}

The main objective of this section is to derive error estimates for $U-u$. With $\tilde{u}$ defined by (3.1), we write the error

$$
U-u=(U-\tilde{u})+(\tilde{u}-u)=\xi+\eta .
$$

The lemmas in $\S 3$ give estimates on $\eta$. It remains to estimate $\xi$. To do this, we need an additional assumption on $U$. Assume that there exists a positive 
constant $c^{*}$ such that

$$
\left\|U_{t}\right\|_{L_{\infty}\left(L_{\infty}\right)} \leq c^{*}
$$

Without loss of generality, assume $c^{*} \geq 2 \tilde{c}$ (see (3.54)).

The following theorem gives the fundamental result on $\xi$.

Theorem 4.1. Let $u, U$, and $\tilde{u}$ be the solutions of problems (2.1), (2.3), and (3.1), respectively. Assume that $u, u_{t} \in L_{2}\left(H^{2}(\Omega)\right) \cap L_{\infty}\left(W^{1, \infty}(\Omega)\right)$ and that $u_{t t} \in L_{2}\left(H^{2}(\Omega)\right)$. Then

$$
\left\|\xi_{t}\right\|_{L_{\infty}\left(L_{2}\right)}+\|\xi\|_{L_{\infty}\left(S_{h}\right)}+\left\|\xi_{t}\right\|_{L_{2}\left(S_{h}\right)} \leq c h^{2} .
$$

Proof. Using (2.1), (2.3), and (3.1), we observe that

$$
\begin{aligned}
\left(\xi_{t}, V\right)+A_{h}(U ; \xi, V)= & -\left(\eta_{t}, V\right)+((a(u)-a(U)) \nabla \tilde{u}, \nabla V)_{h} \\
& +\sum_{i=1}^{2}\left(b_{i}(U) U_{x_{i}}-b_{i}(u) u_{x_{i}}, V\right)_{h} \\
& +(f(U)-f(u), V) \quad \forall V \in S_{h} .
\end{aligned}
$$

Differentiating (4.3) with respect to $t$ yields

$$
\begin{aligned}
\left(\xi_{t t}, V\right) & +A_{h}\left(U ; \xi_{t}, V\right) \\
= & -\left(\eta_{t t}, V\right)-\left((a(U))_{t} \nabla \xi, \nabla V\right)_{h}+\left((a(u)-a(U)) \nabla \tilde{u}_{t}, \nabla V\right)_{h} \\
& +\left((a(u)-a(U))_{t} \nabla \tilde{u}, \nabla V\right)_{h} \\
& +\sum_{i=1}^{2}\left(\left(b_{i}(U) U_{x_{i}}-b_{i}(u) u_{x_{i}}\right)_{t}, V\right)_{h} \\
& +\left((f(U)-f(u))_{t}, V\right) \quad \forall V \in S_{h} .
\end{aligned}
$$

We first discuss estimates of (4.3). Setting $V=\xi_{t}$ and using (4.1), we see that the left-hand side of $(4.3)$ is

$$
\begin{gathered}
\left(\xi_{t}, \xi_{t}\right)+\frac{1}{2} \frac{d}{d t} A_{h}(U ; \xi, \xi)-\left(a_{p}(U) U_{t} \nabla \xi, \nabla \xi\right)_{h} \\
\geq\left\|\xi_{t}\right\|^{2}+\frac{1}{2} \frac{d}{d t} A_{h}(U ; \xi, \xi)-c c^{*}|\xi|_{1, h}^{2}
\end{gathered}
$$

Also,

$$
-\left(\eta_{t}, \xi_{t}\right) \leq\left\|\eta_{t}\right\|^{2}+\left\|\xi_{t}\right\|^{2}
$$

and

$$
\left(f(U)-f(u), \xi_{t}\right) \leq c\left[\|\xi\|^{2}+\|\eta\|^{2}+\left\|\xi_{t}\right\|^{2}\right]
$$


Applying Corollary 3.2 and the inequality $a b \leq a^{2} / 4 \varepsilon+\varepsilon b^{2}$, we get

$$
\left((a(u)-a(U)) \nabla \tilde{u}, \nabla \xi_{t}\right)_{h} \leq c\left(\|\xi\|^{2}+\|\eta\|^{2}\right)+\frac{c_{1}}{12}\left|\xi_{t}\right|_{1, h}^{2} .
$$

Rewrite the third term on the right-hand side of (4.3) as

$$
\sum_{i=1}^{2}\left(b_{i}(U) \xi_{x_{i}}, \xi_{t}\right)_{h}+\sum_{i=1}^{2}\left(\left(b_{i}(U)-b_{i}(u)\right) \tilde{u}_{x_{i}}, \xi_{t}\right)_{h}+\sum_{i=1}^{2}\left(b_{i}(u) \eta_{x_{i}}, \xi_{t}\right)_{h} .
$$

The first two terms of (4.9) are bounded above by

$$
c\left[\|\eta\|^{2}+\|\xi\|^{2}+\left\|\xi_{t}\right\|^{2}+|\xi|_{1, h}^{2}\right]
$$

For the last term of (4.9), we get by Green's formula,

$$
\begin{aligned}
\sum_{i=1}^{2}\left(b_{i}(u) \eta_{x_{i}}, \xi_{t}\right)_{h}= & -\sum_{i=1}^{2}\left(\left(b_{i}(u) \xi_{t}\right)_{x_{i}}, \eta\right)_{h} \\
& +\sum_{i=1}^{2} \sum_{K} \int_{\partial K} b_{i}(u) \eta \xi_{t} N_{i} d s \\
= & Q_{1}+Q_{2} .
\end{aligned}
$$

Obviously,

$$
Q_{1} \leq c\|\eta\|^{2}+\frac{c_{1}}{12}\left|\xi_{t}\right|_{1, h}^{2}
$$

Applying the duality of $H^{1 / 2}(\partial K)$ and $H^{-1 / 2}(\partial K)$ and the trace inequality, we conclude that

$$
\begin{aligned}
Q_{2} & \leq c \sum_{K}\|\eta\|_{-1 / 2, \partial K}\left\|\xi_{t}\right\|_{1 / 2, \partial K} \\
& \leq c \sum_{K}\|\eta\|_{-1 / 2, \partial K}^{2}+\frac{c_{1}}{12}\left(\left\|\xi_{t}\right\|^{2}+\left|\xi_{t}\right|_{1, h}^{2}\right)
\end{aligned}
$$

Combining (4.5)-(4.13) with (4.3), we obtain

$$
\begin{aligned}
\left\|\xi_{t}\right\|^{2} & +\frac{1}{2} \frac{d}{d t} A_{h}(U ; \xi, \xi) \\
\leq c\left(c^{*}+1\right)\left[\|\xi\|^{2}+|\xi|_{1, h}^{2}+\left\|\xi_{t}\right\|^{2}\right. & +\|\eta\|^{2}+\left\|\eta_{t}\right\|^{2} \\
& \left.+\sum_{K}\|\eta\|_{-1 / 2, \partial K}^{2}\right]+\frac{c_{1}}{4}\left|\xi_{t}\right|_{1, h}^{2} .
\end{aligned}
$$


Now we estimate (4.4). Replacing $V$ by $\xi_{t}$ in (4.4), the coerciveness of the bilinear form $A_{h}(U ; \cdot, \cdot)$ implies that the left-hand side of $(4.4)$ is

$$
\geq \frac{1}{2} \frac{d}{d t}\left\|\xi_{t}\right\|^{2}+c_{1}\left|\xi_{t}\right|_{1, h}
$$

As for the fifth term on the right-hand side of (4.4), we write it in the form

$$
\begin{aligned}
\sum_{i=1}^{2}( & \left.\left(b_{i}(U) U_{x_{i}}-b_{i}(u) u_{x_{i}}\right)_{t}, \xi_{t}\right)_{h} \\
= & \sum_{i=1}^{2}\left(b_{i}(U) \xi_{x_{i} t}, \xi_{t}\right)_{h}+\sum_{i=1}^{2}\left(\left(b_{i}(U)-b_{i}(u)\right) \tilde{u}_{x_{i} t}, \xi_{t}\right)_{h} \\
& +\sum_{i=1}^{2}\left(b_{i}(u) \eta_{x_{i} t}, \xi_{t}\right)_{h}+\sum_{i=1}^{2}\left(\left(b_{i}(U)\right)_{t} \xi_{x_{i}}, \xi_{t}\right)_{h} \\
& +\sum_{i=1}^{2}\left(\left(b_{i}(U)-b_{i}(u)\right)_{t} \tilde{u}_{x_{i}}, \xi_{t}\right)_{h}+\sum_{i=1}^{2}\left(\left(b_{i}(u)\right)_{t} \eta_{x_{i}}, \xi_{t}\right)_{h}
\end{aligned}
$$

Using an argument similar to one given in (4.3), we can estimate each term of (4.16). The remaining terms in (4.4) are bounded as before. Following the same analysis that led to (4.14), we obtain

$$
\begin{aligned}
& \frac{1}{2} \frac{d}{d t}\left\|\xi_{t}\right\|^{2}+c_{1}\left|\xi_{t}\right|_{1, h}^{2} \\
& \leq c\left(c^{*}+1\right)\left[\|\xi\|^{2}+|\xi|_{1, h}^{2}+\left\|\xi_{t}\right\|^{2}+\|\eta\|^{2}\right. \\
& \left.\quad+\left\|\eta_{t}\right\|^{2}+\left\|\eta_{t t}\right\|^{2}+\sum_{K}\|\eta\|_{-1 / 2, \partial K}^{2}\right] \\
& +\frac{c_{1}\left|\xi_{t}\right|_{1, h}^{2} .}{}
\end{aligned}
$$

Adding this inequality to (4.14), we see that

$$
\begin{aligned}
& \frac{1}{2} \frac{d}{d t}\left[\left\|\xi_{t}\right\|^{2}+A_{h}(U ; \xi, \xi)\right]+\frac{c_{1}}{2}\left|\xi_{t}\right|_{1, h}^{2} \\
& \leq c\left(c^{*}+1\right)\left[\|\xi\|^{2}+|\xi|_{1, h}^{2}+\left\|\xi_{t}\right\|^{2}+\|\eta\|^{2}+\left\|\eta_{t}\right\|^{2}\right. \\
& \left.+\left\|\eta_{t t}\right\|^{2}+\sum_{K}\|\eta\|_{-1 / 2, \partial K}^{2}\right] .
\end{aligned}
$$

Integrating (4.18) with respect to $t$ and noticing that

$$
\|\xi\|^{2}=\int_{\Omega}\left(\int_{0}^{t} \xi_{t} d s\right)^{2} d x \leq T \int_{0}^{t}\left\|\xi_{t}\right\|^{2} d s,
$$


we have by the initial conditions,

$$
\begin{aligned}
\left\|\xi_{t}\right\|^{2}+|\xi|_{1, h}^{2}+\int_{0}^{t}\left|\xi_{t}\right|_{1, h}^{2} d s & \\
\leq c\left(1+c^{*}\right)\left[\left\|\xi_{t}(0)\right\|^{2}+\int_{0}^{t}\left(\|\eta\|^{2}\right.\right. & +\left\|\eta_{t}\right\|^{2}+\left\|\eta_{t t}\right\|^{2} \\
& \left.+\sum_{K}\|\eta\|_{-1 / 2, \partial K}^{2}\right) d s \\
& \left.+\int_{0}^{t}\left(\left\|\xi_{t}\right\|^{2}+|\xi|_{1, h}^{2}\right) d s\right],
\end{aligned}
$$

which, upon using Gronwall's lemma, gives

$$
\begin{aligned}
& \left\|\xi_{t}\right\|+|\xi|_{1, h}+\left\|\xi_{t}\right\|_{L_{2}\left(0, t ; S_{h}\right)} \\
& \leq c\left(1+c^{*}\right) \exp \left(c\left(1+c^{*}\right) T\right)\left[\left\|\xi_{t}(0)\right\|+\|\eta\|_{L_{2}\left(L_{2}\right)}\right. \\
& +\left\|\eta_{t}\right\|_{L_{2}\left(L_{2}\right)}+\left\|\eta_{t t}\right\|_{L_{2}\left(L_{2}\right)} \\
& \left.+\int_{0}^{T}\left(\sum_{K}\|\eta\|_{-1 / 2, K}^{2}\right)^{1 / 2} d t\right]
\end{aligned}
$$

Setting $t=0$ in (4.3), we obtain

$$
\left(U_{t}(0)-u_{t}(0), V\right)=0 \quad \forall V \in S_{h} .
$$

Hence,

$$
\left\|\xi_{t}(0)\right\|^{2}=\left(\xi_{t}(0), \xi_{t}(0)\right)=-\left(\eta_{t}(0), \xi_{t}(0)\right) \leq\left\|\eta_{t}(0)\right\|\left\|\xi_{t}(0)\right\|
$$

An application of Lemma 3.2 then yields

$$
\left\|\xi_{t}(0)\right\| \leq\left\|\eta_{t}(0)\right\| \leq c h^{2} .
$$

Now Lemmas 3.2-3.6 imply

$$
\left\|\xi_{t}\right\|_{L_{\infty}\left(L_{2}\right)}+\|\xi\|_{L_{\infty}\left(S_{h}\right)}+\left\|\xi_{t}\right\|_{L_{2}\left(S_{h}\right)} \leq c\left(1+c^{*}\right) \exp \left(c\left(1+c^{*}\right) T\right) h^{2}
$$

To complete our argument, we must show that for $h$ sufficiently small, $\left\|U_{t}\right\|_{L_{\infty}\left(L_{\infty}\right)} \leq 2 \tilde{c} \leq c^{*}$. We use the inverse inequality, (3.54), and (4.21) to see that

$$
\begin{aligned}
\left\|U_{t}\right\|_{L_{\infty}\left(L_{\infty}\right)} & \leq\left\|\xi_{t}\right\|_{L_{\infty}\left(L_{\infty}\right)}+\left\|\tilde{u}_{t}\right\|_{L_{\infty}\left(L_{\infty}\right)} \leq \tilde{c}+c h^{-1}\left\|\xi_{t}\right\|_{L_{\infty}\left(L_{2}\right)} \\
& \leq \tilde{c}+c\left(1+c^{*}\right) \exp \left(c\left(1+c^{*}\right) T\right) h .
\end{aligned}
$$

Then clearly, if $h$ is taken sufficiently small,

$$
\left\|U_{t}\right\|_{L_{\infty}\left(L_{\infty}\right)} \leq 2 \tilde{c} \leq c^{*} \text {. }
$$

Hence the constant in $(4.21)$ can be chosen independent of $c^{*}$. 
We are now in a position to prove the main results of this paper. First, by using Lemmas 3.2 and 3.6 and Theorem 4.1, we obtain immediately the following

Theorem 4.2. Let $u$ and $U$ be the solutions of (2.1) and (2.3), respectively. Assume that $u, u_{t t} \in L_{\infty}\left(H^{2}(\Omega)\right) \cap L_{\infty}\left(W^{1, \infty}(\Omega)\right)$ and that $u_{t t} \in L_{2}\left(H^{2}(\Omega)\right)$. Then

(4.22) $\|U-u\|_{L_{\infty}\left(L_{2}\right)}+\left\|U_{t}-u_{t}\right\|_{L_{\infty}\left(L_{2}\right)}+h\left[\|U-u\|_{L_{\infty}\left(S_{h}\right)}+\left\|U_{t}-u_{t}\right\|_{L_{\infty}\left(S_{h}\right)}\right] \leq c h^{2}$.

We now turn to the maximum-norm estimate for the error in the gradient.

Theorem 4.3. In addition to the hypotheses of Theorem 4.1, assume that the domain $\Omega$ is decomposed into rectangular elements. If $u \in L_{\infty}\left(W^{2, \infty}(\Omega)\right)$, then

$$
\|\nabla(U-u)\|_{L_{\infty}\left(L_{\infty}\right)} \leq c h \log (1 / h) .
$$

Proof. Applying Theorem 4.1 and the inverse inequality, we deduce that

$$
\|\nabla \xi\|_{L_{\infty}\left(L_{\infty}\right)} \leq c h^{-1}\|\xi\|_{L_{\infty}\left(S_{h}\right)} \leq c h .
$$

On the other hand, using Lemma 3.3 and the inverse inequality, we have

$$
\begin{aligned}
\|\nabla \eta\|_{L_{\infty}\left(L_{\infty}\right) \leq} \leq & \|\nabla(\tilde{u}-\Pi u)\|_{L_{\infty}\left(L_{\infty}\right)}+\|\nabla(\Pi u-u)\|_{L_{\infty}\left(L_{\infty}\right)} \\
\leq & c h^{-1}\left[\|\eta\|_{L_{\infty}\left(L_{\infty}\right)}+\|u-\Pi u\|_{L_{\infty}\left(L_{\infty}\right)}\right] \\
& +\|\nabla(\Pi u-u)\|_{L_{\infty}\left(L_{\infty}\right)} \\
\leq & \operatorname{chlog}(1 / h) .
\end{aligned}
$$

Inequality (4.23) is now a direct consequence of (4.24)-(4.25).

Finally, we derive a superconvergence order estimate for the gradient.

Theorem 4.4. Under the hypotheses of Theorem 4.1 and Lemma 3.4,

$$
\left\{\frac{1}{N} \sum_{x_{0} \in G}\left|\nabla(U-u)\left(x_{0}\right)\right|^{2}\right\}^{1 / 2} \leq c h^{2} \quad \forall t \in I .
$$

Proof. In view of Lemma 3.4, we only need to estimate $\xi=U-\tilde{u}$. By Theorem 4.1 and the inverse inequality,

$$
\begin{aligned}
\left\{\frac{1}{N} \sum_{x_{0} \in G}\left|\nabla \xi\left(x_{0}\right)\right|^{2}\right\}^{1 / 2} & \leq c\left\{\frac{1}{N} \sum_{K}\|\nabla \xi\|_{L_{\infty}(K)}^{2}\right\}^{1 / 2} \\
& \leq c\left\{\frac{1}{N} \sum_{K} h^{-2}\|\nabla \xi\|_{L_{2}(K)}^{2}\right\}^{1 / 2} \leq c|\xi|_{1, h} \leq c h^{2} .
\end{aligned}
$$

ACKNOWLEDGMENTS

We wish to express our appreciation to Professor Charles Holland for useful comments. 


\section{BIBLIOGRAPHY}

1. J. Douglas, Jr. and T. Dupont, Galerkin methods for parabolic equations, SIAM J. Numer. Anal. 7 (1970), 575-626.

2. P. Lesaint and M. Zlámal, Convergence of the nonconforming Wilson element for arbitrary quadrilateral meshes, Numer. Math. 36 (1980), 35-52.

3. Z. C. Shi, A convergence condition for the quadrilateral Wilson element, Numer. Math. 44 (1984), 349-361.

4. S. M. Shen, $L_{\infty}$ convergence of nonconforming finite element approximations, Math. Numer. Sinica 8 (1986), 225-230.

5. H. Wang, Superconvergence of the Wilson element, J. Shandong Univ. 21 (1986), 89-95.

6. M. F. Wheeler, A priori $L_{2}$ error estimates for Galerkin approximation to parabolic partial differential equations, SIAM J. Numer. Anal. 10 (1973), 723-759.

Department of Mathematics, Bowling Green State University, Bowling Green, Ohio 43403-0221

Department of Mathematics, Shandong University, Jinan, People's Republic of China 